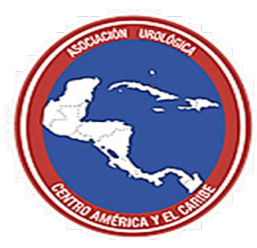

Asociación Urológica de Centroamérica y el Caribe

\title{
TRATAMIENTO ENDOSCÓPICO DE UN TUMOR URETERAL
}

\section{Endoscopic Treatment of a Ureteral Tumor}

\section{Autores}

Michel Hernández Campoalegre ${ }^{(1)}$, Maykel Quintana Rodríguez ${ }^{(1)}$ Jenny María Rodríguez Cabrera ${ }^{(2)}$, Roberto Sánchez Tamaki ${ }^{(3)}$, Mariano Castillo Rodríguez ${ }^{(2)}$ \& Tania González León ${ }^{(1)}$

(1) Centro Nacional de Cirugía de Mínimo Acceso, La Habana, Cuba

(2) Hospital Clínico Quirúrgico "Hermanos Ameijeiras", La Habana, Cuba

(3) Instituto de Nefrología, La Habana, Cuba

Correo electrónico: tania@cce.sld.cu

DOI: https://doi.org/10.54212/27068048.v9i1.17

Esta investigación fue financiada con recursos propios. Declaramos no tener ningún conflicto de intereses

Fecha de envío: 29/04/2021

Fecha de aceptación: 03/06/2021

Fecha de publicación: 30/06/2021

\section{RESUMEN}

OBJETIVO: Presentar el caso clínico de un paciente con diagnóstico de tumor urotelial del uréter al que se le realizó ablación con láser. ANTECEDENTES: El tumor urotelial del tracto urinario superior es una entidad infrecuente. Representa entre el $5-10 \%$ de las neoplasias uroteliales. En las últimas décadas la conservación de la unidad renal ha conferido un papel importante al tratamiento endoscópico de estos tumores en pacientes seleccionados. MATERIAL Y MÉTODOS: Se reporta un paciente de 71 años con un tumor del uréter pélvico. Se hace referencia a los estudios imagenológicos y se muestran, al igual que la cirugía. Se realiza una breve revisión del tema. RESULTADOS: Se trata mediante ureteroscopia con ablación con láser. Evolucionó satisfactoriamente. En el seguimiento a seis meses no se comprueba recurrencia local ni vesical. CONCLUSIONES: La cirugía conservadora de nefronas es una opción en los tumores del urotelio del tracto urinario superior localizados, no músculo-invasivos y de bajo grado. La ablación con láser mediante ureteroscopia es una de sus modalidades cuando se localiza en el uréter distal. Después de este tratamiento los pacientes requieren un seguimiento estricto.

\section{ABSTRACT}

OBJECTIVE: To present the case history of a patient diagnosed with a ureteral urothelial tumor treated with laser ablation. ANTECEDENTS: Upper urinary tract urothelial tumors are a rare entity, representing $5-10 \%$ of urothelial neoplasia. In the past decades preserving the renal unit in selected patients has given endoscopic treatment for these tumors an important role. MATERIAL AND METHODS: A 71 year-old-patient with a pelvic ureteral tumor. Imaging studies are reported and presented, as well as the surgery. A brief revision of the topic is made. RESULTS: The treatment utilized was ureteroscopy with laser ablation. The outcome was satisfactory. No local or vesical recurrence was found in the six- month follow up. CONCLUSIONS: Nephron-sparing surgery is an option for low grade, non-muscle invasive, localized upper urinary tract urothelial tumors. Laser ablation via ureteroscopy is one of the techniques utilized when the tumor is located in the distal ureter. After this treatment, patients require a strict follow-up. 


\section{PALABRAS CLAVE}

urotelio, carcinoma, ureteroscopia, técnicas de ablación, láser

\section{INTRODUCCIÓN}

El carcinoma urotelial es la cuarta neoplasia más común mientras que su localización en el tracto urinario superior es menos frecuente, entre $5-10 \%$. Es más frecuente en varones (3:1) con un pico de incidencia entre los 70-79 años y en el $60 \%$ de los pacientes es infiltrante en el momento del diagnóstico. $(1,2)$

Los tumores del urotelio del tracto urinario superior (TUTUS) son más frecuentes a nivel pielocalicial que ureteral. Puede encontrarse un tumor vesical sincrónico en el $17 \%$ y contralateral en el 2-6\% en el momento del diagnóstico. Se han identificado varios factores de riesgo, entre ellos la exposición al al tabaco. (2)

La nefroureterectomía radical (UN) con la excisión del rodete vesical es el tratamiento estándar. Cuando se trata de tumores de bajo riesgo se debe considerar un tratamiento que conserve la unidad renal como la ablación endoscópica empleando, preferiblemente ureteroscopia flexible o un acceso percutáneo cuando se localiza en el urotelio renal. La resección parcial del uréter es otra opción de tratamiento conservador.

\section{CASO CLÍNICO}

Paciente masculino de 71 años, exfumador, con antecedentes de salud que refirió hematuria total ligera, intermitente por lo que se realizan estudios imagenológicos. El ultrasonido abdominal mostró ligera dilatación del riñón izquierdo.

Se realiza tomografía de abdomen simple y con contraste endovenoso con cortes a $5 \mathrm{~mm}$ la cual informa: hígado de tamaño normal, ho-

\section{KEYWORDS}

urothelium, carcinoma, ureteroscopy, ablation techniques, lasers

mogéneo con imágenes quísticas la mayor de $3 \mathrm{~cm}$ a nivel del segmento 2. Vesícula y vías biliares sin alteraciones. Páncreas pequeño de contornos irregulares. Las glándulas suprarrenales y el bazo sin alteraciones. Ambos riñones de tamaño normal, contornos irregulares por la presencia de múltiples imágenes quísticas de crecimiento exofítico, el mayor de $2 \mathrm{~cm}$ con el aspecto de un quiste simple. A nivel del uréter izquierdo, en su porción pélvica se comprueba defecto de llenado que en su eje longitudinal mide aproximadamente $2 \mathrm{~cm}$ y que impide la progresión del contraste y se acompaña de ligera dilatación del uréter por encima de la lesión. No se identifican alteraciones de la grasa perilesional (Figura 1 A y B). La vejiga no muestra alteraciones. La próstata se identifica aumentada de tamaño con calcificaciones puntiformes en su parte media. Vesículas seminales sin alteraciones. No líquido libre en cavidad abdominal, no se identifican adenopatías. Las estructuras óseas no muestran alteraciones.

La tomografía del tórax no mostró alteraciones.

La cistoscopia fue normal. Previa a la ureteroscopia se realizó ureteropielograma retrógrado que confirmó defecto de llenado a nivel del uréter pélvico. (Figura 1C).

Se realizó ureteroscopia (URS) semirrígida (12 Fr) y se comprobó a nivel del uréter pélvico un tumor pediculado de aproximadamente $2,0 \mathrm{~cm}$ (Figura 2A) y se realizó ablación de su pedículo con láser (Figura 2B). La lesión se extrajo empleando una dormia. Se colocó catéter doble $\mathrm{J}$ y sonda uretral $18 \mathrm{ch}$.

El patólogo confirmó el recibo de una lesión rotulada como tumor urotelial del uréter pélvico de $1,8 \mathrm{~cm}$, que describió microscópicamente como sigue: se observan células uroteliales 
con atipia nuclear mínima, presencia de núcleos hipercromáticos y escasa presencia de mitosis. Por lo que se concluyó como un carcinoma urotelial de bajo grado.

A los 21 días se retiró el catéter doble J, se repitió URS y se comprobó una minúscula lesión de aproximadamente $3 \mathrm{~mm}$ localizada a nivel del sitio tratado, que se extrajo íntegramente con pinza de biopsia y su base fue electrofulgurada (empleando energía monopolar). La cual se concluyó el patólogo concluyó como ureteritis crónica moderada con elementos de fibrosis y descartó la presencia de malignidad. En esa ocasión se dejó un catéter interno-externo que se retiró al día siguiente.

No se produjeron complicaciones en relación con el tratamiento endoscópico.

El paciente se ha seguido durante seis meses, hasta el momento en que se reporta. Está asintomático. Se realizaron TAC contrastada del tracto urinario y URS a los tres y seis meses, respectivamente, después del tratamiento. Se confirmó integridad del uréter y ausencia de recurrencia local como muestran las Figura 3 A y $B$.

\section{DISCUSIÓN}

Aunque el gold standar para los TUTUS ha sido la NU con excisión del rodete vesical distal ipsilateral, en las últimas dos décadas ha cambiado gracias al desarrollo tecnológico en el campo de la endoscopia y la mejor comprensión de los procesos biológicos de la enfermedad. En la actualidad se reconoce la cirugía conservadora de nefronas (CCN) como una opción terapéutica, que incluye el abordaje endoscópico mediante ureteroscopia o percutáneo, la ureterectomía distal y menos frecuentemente la nefrectomía parcial. $(3,4)$

A la NU se le atribuyen beneficios oncológicos, pero está asociada a la morbilidad en relación con los eventos cardiovasculares que se desencadenan ante el daño renal por la pérdida del órgano. Por otro lado, se ha encontrado que el $56 \%$ de los TUTUS presentan un estadio pT1-0, después de una NU. De ahí la importancia que ha recuperado la $\mathrm{CCN}$ en el tratamiento de los TUTUS, en la actualidad. (4)

La CCN para el tratamiento de los TUTUS se indica cuando la enfermedad es localizada y el tumor es de bajo grado, aún en presencia de un riñón contralateral normal o en pacientes seleccionados con severo daño de la función renal, en riñones únicos anatómicos o funcionales, cuando la enfermedad es bilateral, se presenta en pacientes con estados patológicos que implican alto riesgo de recurrencia contralateral (nefropatia balcánica, syndrome de Lynch) o en presencia de comorbilidades que no permitan la realización de la NU. Otros autores la recominedan como la primera línea de tratamiento en los tumores de bajo riesgo, unificales , >1 cm, de bajo grado por citología o biopsia y no invasivos definido por tomografía contrastada del tracto urinario (TAC). $(2,5)$

La evaluación diagnóstica es crucial para la adecuada selección de los pacientes candidatos a CCN. La TAC contrastada es la modalidad de diagnóstico por imágenes por excelencia, puede diagnosticar tumores uroteliales mayores de $5 \mathrm{~mm}$. La resonancia magnética es útil cuando está contraindicado el empleo de contraste iodado. La citología es el gold estándar y su sensibilidad aumenta cuando la muestra se toma directamente de la unidad renal afectada. La URS es el método diagnóstico para la visualización directa de la lesión y la toma de biopsia. En la actualidad nuevas técnicas de imagen endoscópica han sido desarrolladas como el sistema IMAGE $1 \mathrm{~S}$ de Storz, las imágenes de banda estrecha (NBI) y la fotodinamia diagnóstica. La biopsia tomada con el empleo de pinzas o cesta permite confirmar el diagnostico y el grado del tumor. Para la evaluación de estos pacientes también pueden emplearse biomarcadores, pero aún es controversial; y emergen otros elementos diagnósticos como la la tomografía de coherencia óptica y la endomicroscopía láser confocal, pero requieren de estudios futuros para su mejor recomendación. $(3,6)$ 
En el caso que se presenta no contamos con disponiblidad técnica para la realización de la citología urinaria. La TAC contrastada y la URS fueron decisivas para el diagnóstico.

La evolución técnica de la endoscopia así como las innovaciones en la tecnología óptica, digital y en el campo del láser ha permitido la mejor visualización del los TUTUS y la efectividad de su tratamiento endoscópico. El refinamiento de los instrumentos para biopsias endocópicas ha permitido mejor calidad de los especímenes obtenidos lo que ha mejorado el estadiamiento histopatológico, pero aún existen limitaciones.(4)

La URS semirrígida puede ser empleada en el tratamiento de los tumores localizados en el uréter distal y medio, mientras que para lesiones del uréter proximal, la pelvis y los cálices se recomienda la URS flexible y el empleo de camisetas de acceso para evitar mayor trauma. Las biopsias se pueden obtener con pinzas de biopsia, cestas para litiasis, pinzas de agarre o por cepillado. Después de la biopsia, el láser se emplea para lograr la hemostasia, la resección del tumor y la fulguración del lecho tumoral. Tradicionalmente, se han empleado el YAG o el holmium láser. En el paciente que se presenta se empleó la URS semirrígida y láser holmiun de la firma Storz. $(4,7)$

También se ha descrito el empleo de resección y fulguración con electrocauterio a baja intensidad y en áreas pequeñas, para lo cual se ha empleado un asa similar a la de la resección transuretral de tumores vesicales, pero no está fácimente disponible en el mercado. En el caso que se presenta empleamos un electrodo monopolar para electrofulgurar la base de la pequeña lesión que se encontró en la segunda URS. (4)

Debido a la recurrencia después de la CCN en los TUTUS y extrapolando los beneficios en los tumores uroteliales vesicales, se ha recomendado el empleo de terapia adyuvante local, fundamentalmente, con quimiterapia (Mitomicin C). Sin embargo, todavía es controversial la forma óptima de instilación y los beneficios oncológicos no son del todo claros, aunque la evidencia actual indica que la adyuvancia con Mitomicin C es factible y bien tolerada. En este paciente no se empleó terapia adyuvante, por no disponer. (3)

Aún las tasas de recurrencias después de la CCN son altas por lo que resulta muy importante un estricto régimen de seguimiento que se recomienda, en el caso de los tumores de bajo grado, mediante cistoscopia, citología de orina, tomografía y URS a los 3 y 6 meses inicialmente y después anualmente para cambiar la conducta a NU si la enfermedad migra a un alto riesgo. (2)

En general, se reportan tasas de $65 \%$ y $44 \%$ de recidivas intravesicales, respectivamente, y $33 \%$ de necesidad de practicar NU o ureterectomía, después del tratamiento endoscópico de los TUTUS. Otros autores comentan tasas de recurrencia entre $19-21 \%$ y de NU de recate en los tumores de bajo grado de $16,7 \%$ y $28,6 \%$ en los de alto grado. $(4,5)$

Hasta los primeros seis meses el paciente que se reporta no ha presentado recurrencia local, ni vesical.

El tratamiento de TUTUS parecen estar cambiando hacia el manejo quirúrgico conservador con ablación del tumor y aumentando el uso de quimioterapia neoadyuvante. Se están realizando más biopsias de tumores primarios, probablemente reflejando mejores instrumentos y entrenamiento ureteroscópicos.(8)

Conclusiones: La cirugía conservadora de nefronas es una opción en los tumores del urotelio del tracto urinario superior localizados, no músculo-invasivos y de bajo grado. La ablación con láser mediante ureteroscopia es una de sus modalidades cuando se localiza en el uréter distal. Después de este tratamiento los pacientes requieren un seguimiento estricto. 


\section{REFERENCIAS}

1. Siegel RL, Miller KD, Jemal A. Cancer statistics, 2019. CA Cancer J Clin. 2019 Jan;69(1):734. https://doi.org/10.3322/caac.21551

2. Rouprêt $M$, Babjuk $M$, Burger $M$ et al. EAU Guidelines Office, Arnhem, The Netherlands. http://uroweb.org/guidelines/compilations-of-all-guidelines/

3. Territo A, Foerster B, Shariat SF et al. Diagnosis and kidney-sparing treatments for upper tract urothelial carcinoma: state of the art. Minerva Urol Nefrol. 2018;70(3):242-251.

https://doi.org/10.23736/S0393-2249.18.03058-8

4. Petros FG, Li R, Matin SF. Endoscopic approaches to upper tract urothelial carcinoma. Urol Clin North Am. 2018 May;45(2):267286. https://doi.org/10.1016/j.ucl.2017.12.009

5. Metcalf M, Pierorazio PM. Future strategies to enhance kidney preservation in upper urinary tract urothelial carcinoma. Transl Androl Urol. 2020 Aug;9(4):1831-1840.

https://doi.org/10.21037/tau.2019.11.09
6. Azizi M, Cheriyan SK, Peyton CC et al. Optimal management of upper tract urothelial carcinoma: an unmet need. Curr Treat Options Oncol. 2019 Apr 1;20(5):40.

https://doi.org/10.1007/s11864-019-0637-2

7. Scotland KB, Kleinmann N, Cason D et al. Ureteroscopic management of large $\geq 2 \mathrm{~cm}$ upper tract urothelial carcinoma: A comprehensive 23-year experience. Urology. 2018; 121:66-73.

https://doi.org/10.1016/j.urology.2018.05.042

8. Browne BM, Stensland KD, Moynihan MJ et al. An analysis of staging and treatment trends for upper tract urothelial carcinoma in the national cancer database. Clin Genitourin Cancer. 2018 Aug;16(4): e743-e750.

https://doi.org/10.1016/j.clgc.2018.01.015

Copyright (c) 2021 Michel Hernández Campoalegre, Maykel Quintana Rodríguez, Jenny María Rodríguez Cabrera, Roberto Sánchez Tamaki, Mariano Castillo Rodríguez \& Tania González León

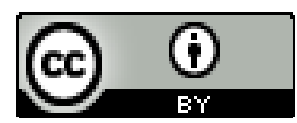

Este texto está protegido por una licencia Creative Commons 4.0.

Usted es libre para Compartir - copiar y redistribuir el material en cualquier medio o formato - y Adaptar el documento — remezclar, transformar y crear a partir del material— para cualquier propósito, , incluso para fines comerciales, siempre que cumpla la condición de:

Atribución: Usted debe dar crédito a la obra original de manera adecuada, proporcionar un enlace a la licencia, e indicar si se han realizado cambios. Puede hacerlo en cualquier forma razonable, pero no de forma tal que sugiera que tiene el apoyo del licenciante o lo recibe por el uso que hace de la obra. 